EPJ Web of Conferences 38, 08002 (2012)

DOI: $10.1051 /$ epjconf/20123808002

(c) Owned by the authors, published by EDP Sciences, 2012

\title{
The deformation energy and fission barriers of heavy nuclei
}

\author{
F.A.Ivanyuk ${ }^{\mathrm{a}}$ \\ Institute for Nuclear Research, Kiev, Ukraine
}

\begin{abstract}
The macroscopic part of the fission barriers of medium and heavy nuclei is calculated looking for the minimum of liquid drop energy at fixed volume and elongation without using any shape parameterization. The fission barrier heights, obtained within the Lublin-Strasbourg Drop model, are approximated by a simple analytical expression as functions of $Z$ and $(N-Z) / A$ only. In addition, using the topological theorem by Myers and Świątecki which allows to represent the barrier height as the sum of the ground state microscopic correction (which is calculated and tabulated for all nuclei) and the liquid-drop fission barrier, we propose a simple but quite accurate approximation of the fission barrier heights.
\end{abstract}

\section{Introduction}

In the theory of nuclear fission or fusion-fission reactions one often introduces the shape degrees of freedom (deformation parameters) as the dynamical variables. Usually, the few deformation parameters are used and the variation of shape is restricted to a certain class of shapes.

In past a lot of different shape parametrization was introduced. In all these cases the question of the completeness of the chosen class of shapes arises.

Another problem appears in the description of fusionfission reactions based on Langevin equation. There one often starts the dynamical treatment from so called contact point (which is not strictly defined) or uses different parameterizations for the approaching stage and evolution of the bound system. The transition from divided to bound system is not well defined and is a source of a considerable uncertainty of calculated results.

It is highly desirable to develop a method which would not rely on any shape parameterization and would be able to describe the transformation of the shape from two fragments to the mono-system. A step in this direction is described in the present talk.

\section{The variational principle for liquid drop shapes}

A possibility to specify the shape of the nuclear surface which would not rely on a shape parameterization was proposed by V. Strutinsky [1] in 1963. It was suggested in [1] to define the profile function looking for the minimum of the liquid-drop energy, $E_{\mathrm{LD}}=E_{\text {surf }}+E_{\mathrm{Coul}}$, under the constraint that the volume $V$ and the elongation $R_{12}$ are fixed

$$
\frac{\delta}{\delta \rho}\left(E_{\mathrm{LD}}-\lambda_{1} V-\lambda_{2} R_{12}\right)=0,
$$

where $\lambda_{1}$ and $\lambda_{2}$ are the corresponding Lagrange multipliers. In (1) $\rho(z)$ is the profile function by rotation of which

\footnotetext{
a e-mail: ivanyuk@kinr.kiev.ua
}

around $z$-axis one gets the shape of nucleus (in case of axial symmetry). The elongation parameter $R_{12}$ was chosen in Ref. [1] as the distance between the centers of mass of the left and right parts of the nucleus

$$
R_{12}=\frac{2 \pi}{V} \int_{z_{1}}^{z_{2}} \rho^{2}(z)|z| d z, \quad V=\pi \int_{z_{1}}^{z_{2}} \rho^{2}(z) d z
$$

The minimization of $E_{\mathrm{LD}}-\lambda_{1} V-\lambda_{2} R_{12}$ with respect to the variation of the profile function $\rho(z)$ leads to an integrodifferential equation for $\rho(z)$

$$
\rho \rho^{\prime \prime}=1+\left(\rho^{\prime}\right)^{2}-\rho\left[\lambda_{1}+\lambda_{2}|z|-10 x_{\mathrm{LD}} \Phi_{S}\right]\left[1+\left(\rho^{\prime}\right)^{2}\right]^{\frac{3}{2}} .
$$

Here $\Phi_{S} \equiv \Phi(z, \rho(z))$ is the Coulomb potential at the nuclear surface, and $x_{\mathrm{LD}}$ is the fissility parameter of the liquid drop [2]

$$
x_{\mathrm{LD}} \equiv E_{\mathrm{Coul}}^{(0)} / 2 E_{\mathrm{surf}}^{(0)}=\frac{3}{10} \frac{Z^{2} e^{2}}{4 \pi R_{0}^{3} \sigma},
$$

where $\sigma$ is the surface tension coefficient. In (4) and everywhere below the index ${ }^{(0)}$ refers to the spherical shape.

By solving Eq. (3) for given $x_{L D}$ one obtains the profile function $\rho(z)$. The sequence of shapes (we call them

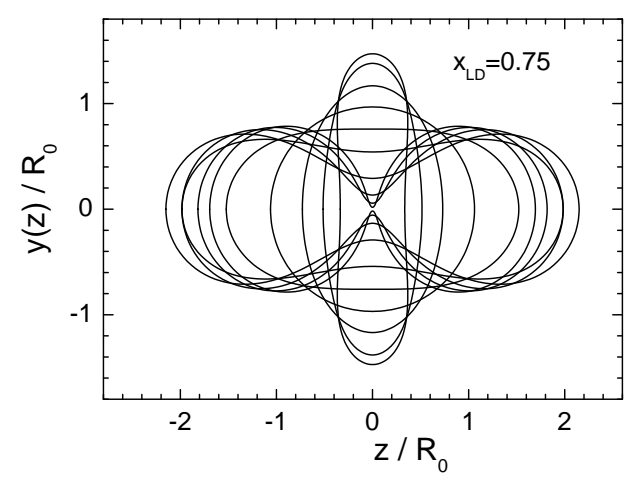

Fig. 1. The solutions of Eq. (3) corresponding to different values of Lagrange multiplier $\lambda_{2}$ which fixes the deformation $R_{12}$.

This is an Open Access article distributed under the terms of the Creative Commons Attribution License 2.0, which permits unrestricted use, distribution, and reproduction in any medium, provided the original work is properly cited. 
optimal shapes) obtained for different values of the constraining parameter $\lambda_{2}$ is shown in figure 1. Varying the parameter $\lambda_{2}\left(\lambda_{1}\right.$ is fixed by the volume conservation condition) one obtains the shapes ranging from a very oblate shape (disk) up to two touching spheres.

The deformation energy $E_{\mathrm{def}}^{L D}=E_{\mathrm{LD}}-E_{\mathrm{LD}}^{(0)}$ (in units of the surface energy for spherical shape)

$$
E_{\mathrm{def}}^{L D} / E_{\mathrm{surf}}^{(0)}=B_{\text {surf }}-1+2 x_{\mathrm{LD}}\left(B_{\mathrm{Coul}}-1\right)
$$

where $B_{\text {Coul }} \equiv E_{\text {Coul }} / E_{\text {Coul }}^{(0)}$ and $B_{\text {surf }} \equiv E_{\text {surf }} / E_{\text {surf }}^{(0)}$, calculated for the shapes shown in figure 1 , is presented in figure 2 .

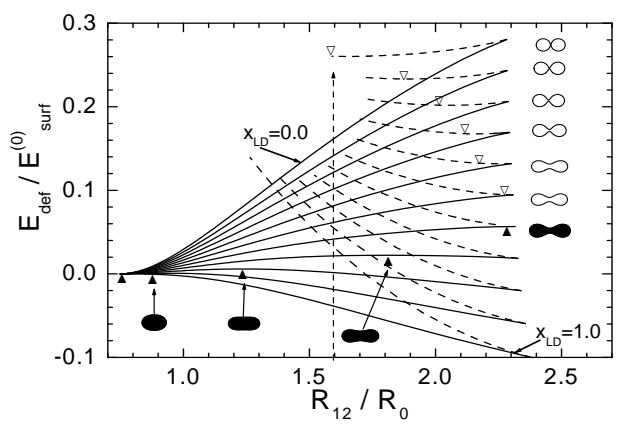

Fig. 2. Liquid-drop deformation energy (5) as function of parameter $R_{12}$ for a few values of the fissility parameter $x_{\mathrm{LD}}$.

One can see from figure 2 that the elongation $R_{12}$ of the shapes shown in these figures is limited by some maximal value $R_{12}^{\max }$. This critical deformation was interpreted in [1] as the scission point. Note, that at the scission the neck radius is still rather large, the neck radius at the critical deformation is approximately equal to $(0.25-0.30) R_{0}$ for a fissility parameter in the range $0.4 \leq x_{L D} \leq 0.9$ which indicates that the nucleus breaks apart at a finite neck radius.

Another peculiarity of figure 2 is the upper branch of the deformation energy at large deformation. Along this branch the neck of the drop becomes smaller and smaller until the shape turns into the two touching spheres. It turns out, however, that the upper branch of the deformation energy corresponds not to the minimum of $E_{L D}$ but to its maximum (the condition (1) holds true both for the minimum and maximum of $E_{L D}$ ). This can be easily verified by adding to the optimal profile function $\rho(z)$ an arbitrary small perturbation $\delta \rho(z)$ and calculating the deformation energy with perturbed profile function $\rho(z)+\delta \rho(z)$. Thus, the upper branch of $E_{d e f}$ corresponds to the ridge of the potential energy surface in the coordinates elongation and the neck thickness.

Having the solutions of the variational problem (3), one can immediately check the quality of various shape parameterizations. In figure 3 the deformation energy calculated with the optimal shapes are compared with that obtained within the Cassini parameterization [3]. The Cassini ovaloids reproduce the shape of fissioning nuclei rather well almost up to the saddle. After the saddle the additional deformation parameters should be taken into account.

In the same way one can check the accuracy of any other shape parameterization.

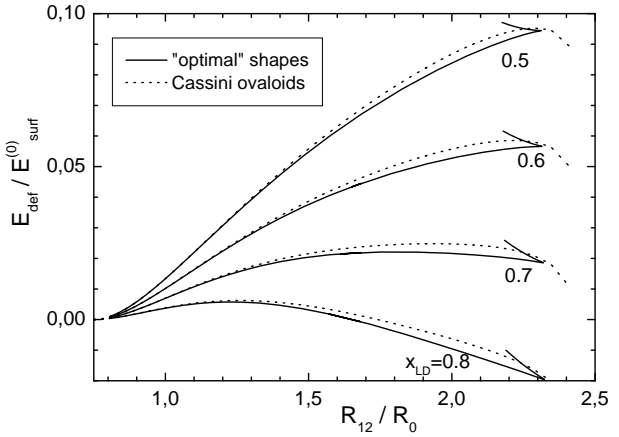

Fig. 3. The comparison of the deformation energy calculated with the optimal shapes and the Cassini ovaloids.

\section{Mass-asymmetric shapes}

The above formalism can be generalized to mass-asymmetric shapes. For this purpose one has to add one more constraint fixing the left-right asymmetry. Commonly the mass asymmetry is understood as the ratio $\delta \equiv\left(M_{L}-\right.$ $\left.M_{R}\right) /\left(M_{L}+M_{R}\right)$, where $M_{L}$ and $M_{R}$ are the masses of the left and right parts of nucleus. For shapes with neck, it is the neck that divides the shape into the left and right parts. For pear-like shapes a neck does not exist and one has to introduce another quantity which would divide the shape into left and right parts.

For this aim let us look at the local curvature of the surface $H(z)$,

$$
H(z)=\frac{1}{2}\left(\frac{1}{R_{1}}+\frac{1}{R_{2}}\right),
$$

with $R_{1}$ and $R_{2}$ being the local principal radii of curvature. In the case of axially symmetric shapes the radii $R_{1}$ and $R_{2}$ are expressed in terms of the profile function

$$
R_{1}=\rho(z) \sqrt{1+\left(\rho^{\prime}\right)^{2}}, \quad R_{2}=-\left[1+\left(\rho^{\prime}\right)^{2}\right]^{\frac{3}{2}} / \rho^{\prime \prime}
$$

On any surface one can always find a place, where the curvature is the largest. Let $z^{*}$ be that place. In case of leftright symmetric shapes $z^{*}$ would coincide with the center of mass, $z_{c m}=z^{*}$. For mass asymmetric shapes $z_{c m} \neq z^{*}$ and the difference $z_{c m}-z^{*}$ can be considered as a measure of the mass asymmetry. Formally $z_{c m}-z^{*}$ can be written as

$$
z_{c m}-z^{*}=\frac{\pi}{V} \int_{z_{1}}^{z_{2}}\left(z-z^{*}\right) \rho^{2}(z) d z
$$

what can be included as an additional constraint in the form $-\lambda_{3}\left(z_{c m}-z^{*}\right)$ into Eq. (1) leading to the following modification of equation (3):

$$
\begin{aligned}
\rho \rho^{\prime \prime} & =1+\left(\rho^{\prime}\right)^{2}-\rho\left[\lambda_{1}+\lambda_{2}\left|z-z^{*}\right|+\lambda_{3}\left(z-z^{*}\right)\right. \\
& \left.-10 x_{\mathrm{LD}} \Phi_{S}\right]\left[1+\left(\rho^{\prime}\right)^{2}\right]^{\frac{3}{2}} .
\end{aligned}
$$

The equation (9) can be solved in the same way as (3). Having the solutions of Eq. (9) at ones disposal one can examine the dependence of the saddle or scission configuration on the mass asymmetry. 


\section{The scission configuration}

The knowledge about the scission shape and energy is of the prime importance for the description of kinetic energy of the fission fragments or number of neutrons emitted during the fission process. The total kinetic energy of fission fragments and the number of emitted neutrons are among the most important observables of fission experiments. Comparing the experimental results with the theoretical predictions one can judge how correct is our understanding of the fission process.

The existing dynamical codes for the fission process (based on the Langevin equations for the shape degrees of freedom) can, in principle, calculate the number of evaporated particles during the evolution from the ground state to the scission point. There is no dynamical theory, to the best of our knowledge, for the evaporation of gammas or light particles from the fission fragments. Instead one usually uses the considerations of energy conservation. If one knows the excitation energy "just before the scission" and the final (ground state) energy of the fission fragments, one can get the energy which can be taken away by neutrons. The number of emitted neutrons is estimated then as the available excitation energy divided by the neutron binding energy.

To calculate the ground state energy of the fission fragment is relatively easy. Usually one would apply the macroscopic-microscopic method, i.e. would calculate the liquid drop type of energy plus Strutinsky's shell correction. Then the minimization with respect to the shape should be carried out.

The calculation of the excitation energy "just before the scission" is somewhat more complicated. First of all one should have a reliable information on the shape of the nucleus at the scission point. For this purpose one could apply the method described above. The liquid drop energy for the mass asymmetric optimal shapes is plotted in figure 4. Now instead of scission point one has a scission line the deformation above which the compact shapes do not exist.

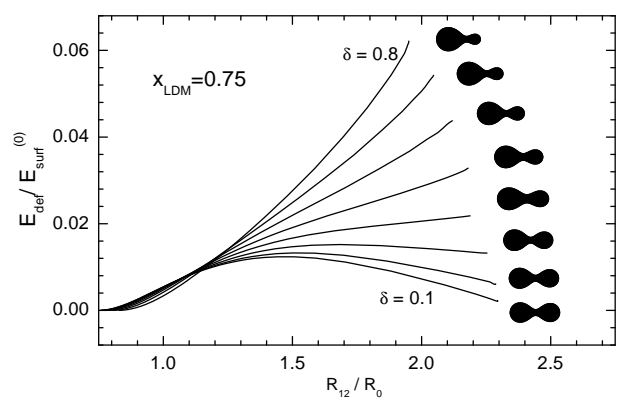

Fig. 4. The liquid drop deformation energy (5) as function of the elongation $R_{12}$ for few values of mass asymmetry parameter $\delta$.

The optimal shapes along the scission line are collected in figure 5. An interesting feature of this plot is the fact that the neck radius $R_{n e c k}^{(s c)}$ of the optimal shape along the scission line does not depend much on the mass asymmetry, $R_{\text {neck }}^{(s c)} \approx 0.2 R_{0}$. Having the scission shapes at ones disposal one can calculate the different quantities of interest like the Coulomb energy at the scission point, the excitation energy, etc.

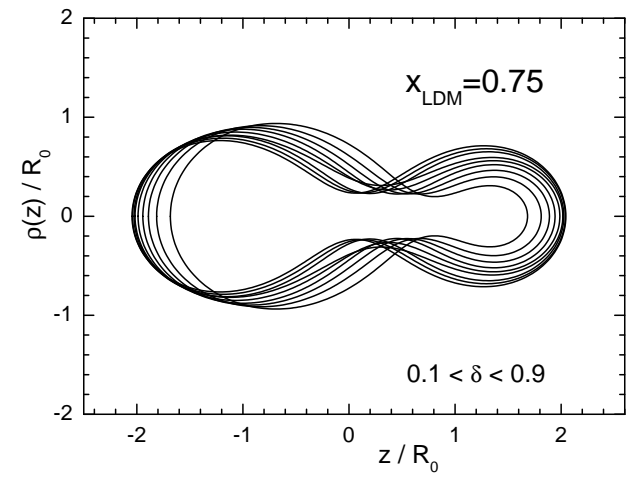

Fig. 5. The shape of nucleus along the scission line for the fissility parameter $x_{L D}=0.75$.

\section{Applications: the barriers of heavy nuclei}

As an application of the presented above approach the fission barrier heights of heavy nuclei were calculated [4]. In order to be able to compare the calculate results with the experimental data besides the liquid drop energies the surface curvature energy term was included into the liquid drop model and we took into account the shell corrections. The curvature energy appears in the leptodermous expansion $[5,6]$, according to which the energy of a nucleus in a Thomas-Fermi type approximation consists of volume, surface, curvature and Gauss curvature terms,

$$
E_{\mathrm{TF}}=E_{\mathrm{vol}}+E_{\mathrm{surf}}+E_{\mathrm{curv}}+E_{\mathrm{Gcurv}} .
$$

Each term on the r.h.s of (10) is smaller by a factor $A^{1 / 3}$ as compared with the previous one. The Gauss curvature term, $E_{\text {Gcurv }} \propto A^{0}$, is the smallest one and can be neglected since it does not depend on deformation. The curvature energy $E_{\text {curv }}$ is expressed in terms of the local curvature $H(z)$, see (6),

$$
E_{\text {curv }}=\frac{E_{\text {curv }}^{(0)}}{4 \pi R_{0}} \int H(z) d S,
$$

where $E_{\text {curv }}^{(0)}$ is the curvature energy at spherical shape. Formally, $E_{\text {curv }}$ can be combined with the surface energy

$$
E_{\text {surf }}+E_{\text {curv }}=\sigma \int[1+\Gamma H(z)] d S,
$$

where $\Gamma$ is given by the ratio of curvature and surface energies at spherical shape

$$
\Gamma / R_{0} \equiv E_{\text {curv }}^{(0)} / E_{\text {surf }}^{(0)} .
$$

The replacement of $E_{\text {surf }}$ in (1) by (12) leads to the following modification of Eq. (3):

$$
\begin{aligned}
& \rho \rho^{\prime \prime}\left[1+\Gamma / \sqrt{\rho^{2}+\left(\rho \rho^{\prime}\right)^{2}}\right]= \\
& 1+\left(\rho^{\prime}\right)^{2}-\rho\left[\lambda_{1}+\lambda_{2}|z|-10 x_{\mathrm{LD}} \Phi_{S}\right]\left[1+\left(\rho^{\prime}\right)^{2}\right]^{\frac{3}{2}} .
\end{aligned}
$$

Eq. (14) is solved in the same way as (3). A few examples of the deformation energy

$$
E_{\mathrm{def}}^{\mathrm{LD}}=E_{\mathrm{Coul}}^{(0)}\left(B_{C}-1\right)+E_{\text {surf }}^{(0)}\left(B_{S}-1\right)+E_{\text {curv }}^{(0)}\left(B_{K}-1\right),
$$

with $B_{K} \equiv E_{\text {curv }} / E_{\text {curv }}^{(0)}$, calculated with the profile function obtained from (14) are shown in figure 6. From the results 
of these calculations it follows that for $\Gamma \approx \pm(0.05-0.10)$ the change of the shape due to the curvature correction is very small. At the same time, the correction to the energy given by (11) leads to a noticeable shift of the saddle point deformations and energies.

Consequently, taking the curvature energy into account can change considerably the coefficients of the fitting formula for the nuclear masses or binding energies.

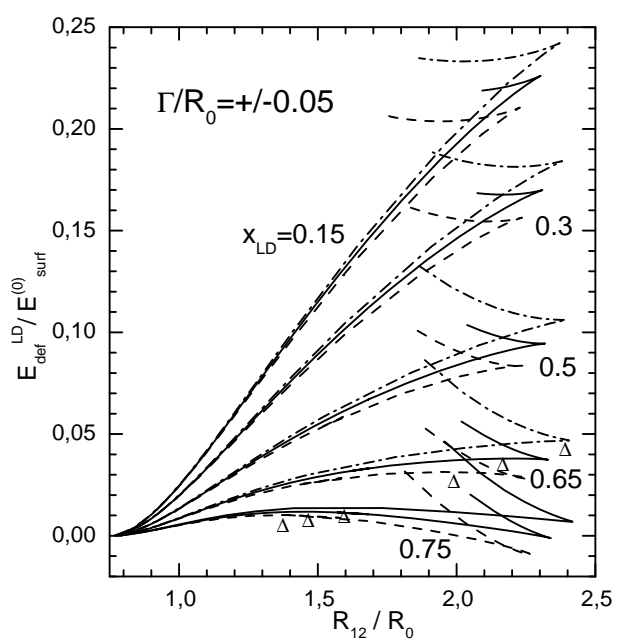

Fig. 6. Deformation energy (15) for a few values of the fissility parameter (indicated in figure). The solid, dash-dotted and dash lines correspond to $\Gamma / R_{0}=0,+0.05$ and -0.05 . The saddle point deformations are indicated by $\boldsymbol{\Lambda}$.

In 1963 the influence of the surface curvature term on the nuclear shape and deformation energy was studied by Strutinsky [7]. At that time it was not clear what should be the value of $\Gamma$, so that two options were considered for $\Gamma$, namely $\Gamma / R_{0}= \pm 0.1$. The curvature term was studied later in [8], where its magnitude was adjusted to the experimental fission barrier heights known at that time. A recent fit of the nuclear ground state masses on the base of the fitting expression for the liquid drop type macroscopic energy which includes explicitly the curvature energy was carried out in [9]. The microscopic, odd-even and congruence energy corrections which one has to add to the macroscopic energy were taken from [10], where a realistic Thomas-Fermi model was developed, which describes the masses of known nuclei with high accuracy

$$
\begin{aligned}
& M(Z, N ; \text { def })=Z M_{\mathrm{H}}+N M_{\mathrm{n}}-0.00001433 Z^{2.39} \\
& \quad+E_{\mathrm{LSD}}(Z, N ; \text { def })+E_{\mathrm{micr}}(Z, N ; \text { def })+E_{\mathrm{o}-\mathrm{e}}+E_{\mathrm{cong}}
\end{aligned}
$$

with

$$
\begin{aligned}
E_{\mathrm{LSD}} & =b_{\mathrm{vol}}\left(1-\kappa_{\mathrm{vol}} I^{2}\right) A+b_{\text {surf }}\left(1-\kappa_{\mathrm{surf}} I^{2}\right) A^{\frac{2}{3}} B_{S}(\mathrm{def}) \\
& +b_{\text {curv }}\left(1-\kappa_{\mathrm{curv}} I^{2}\right) A^{\frac{1}{3}} B_{K}(\mathrm{def}) \\
& +\frac{3}{5} \frac{Z^{2} e^{2}}{r_{0}^{\mathrm{ch}} A^{1 / 3}} B_{C}(\mathrm{def})-C_{4} \frac{Z^{2}}{A} .
\end{aligned}
$$

In (17) $I \equiv(N-Z) / A$ and $E_{\text {micr }}$ is the microscopic energy containing the contributions from the shell, the pairing and the ground state deformation effects

$$
E_{\text {micr }}=\delta E_{\text {shell }}+\delta E_{\text {pair }}+\delta E_{\text {def }} .
$$

Here $\delta E_{\text {def }}$ is equal to the difference of the nuclear macroscopic energy at the ground state deformation and the macroscopic energy of the spherical nucleus. The term $E_{\mathrm{O}-\mathrm{e}}$ describes odd-even energy (for details confer to Ref. [10]) and, according to [10], the congruence energy $E_{\text {cong }}$ is

$$
E_{\text {cong }}=-10 \exp (-4.2|I|) \mathrm{MeV} .
$$

In Ref. [9] the parameters of the mass formula (16), (17) were adjusted to the nuclear binding energies of $2766 \mathrm{nu}-$ clei with $Z \geq 8$ and $N \geq 8$ [11] known at that time. The obtained set of parameters is given by

$$
\begin{gathered}
b_{\mathrm{vol}}=-15.4920 \mathrm{MeV}, \kappa_{\mathrm{vol}}=1.8601, \\
b_{\text {surf }}=16.9707 \mathrm{MeV}, \kappa_{\text {surf }}=2.2938, \\
b_{\text {curv }}=3.8602 \mathrm{MeV}, \quad \kappa_{\text {curv }}=-2.3764, \\
r_{0}^{\text {ch }}=1.21725 \mathrm{fm}, \quad C_{4}=0.9181 .
\end{gathered}
$$

The rms deviation of the theoretical binding energies from the experimental values was found [9] to be equal to 0.698 $\mathrm{MeV}$ only, while the mean-squared-deviation of the barrier heights $\left\langle\delta V_{B}\right\rangle=3.56 \mathrm{MeV}$, which decreases to only $0.88 \mathrm{MeV}$ when the four lightest nuclei (with $A \approx 100$ ) are disregarded i.e., when only the nuclei with $Z>70$ are considered. Note, than in [9] the parameters of (17) were fitted to the nuclear masses and not to the fission barriers. One has to mention that the heights of the LSD fission barriers approach significantly to the measured values for light nuclei when the deformation dependence of the congruence energy proposed in Ref. [12] is taken into account [13].

In the calculation of the deformation energy in [13] the shape of the surface was defined by the expansion around the spherical shape in terms of Legendre polynomials. All even multipoles $\beta_{\lambda}$ with $\lambda \leq 14$ were taken into account and the minimization of the energy with respect to all $\beta_{\lambda}$ at fixed $\beta_{2}$ was carried out.

It is of a certain interest to check whether the fission barriers would change if one would calculate the energy with the optimal shapes (14) which satisfy exactly the condition of minimal liquid drop energy. For this we have calculated the deformation energy

$$
E_{d e f}^{\mathrm{LSD}}=E_{\mathrm{LSD}}(\mathrm{def})-E_{\mathrm{LSD}}^{(0)}
$$

Typical examples of the deformation energy (21) for medium and heavy nuclei are shown in figure 7 . In this calculation the charge asymmetry $(N-Z) / A$ was fixed to be equal to 0.26 , what corresponds to the isotopes with the highest fission barrier, see figure 7 .

The maximal value of the deformation energy (21) in figure 7 defines the macroscopic part of the fission barrier $B_{\mathrm{LSD}}$. The results of numerical calculations for $B_{\mathrm{LSD}}$ are shown in figure 8 . For practical purposes an analytical approximation for the $B_{\mathrm{LSD}}$ would be very useful. It turns out that for fixed $Z$ the dependence of $B_{\mathrm{LSD}}$ on the charge asymmetry parameter $I$ is surprisingly well approximated by a Gaussian

$$
B_{\mathrm{LSD}}(Z, I)=B_{\max }(Z) \exp \left[-\left(\frac{\left.I-I_{0}(Z)\right)}{\Delta I(Z)}\right)^{2}\right] .
$$

The dependence of $B_{\max }, I_{0}$ and $\Delta I$ on $Z$ is smooth but a little bit complicated. We did not manage to find a simple and 


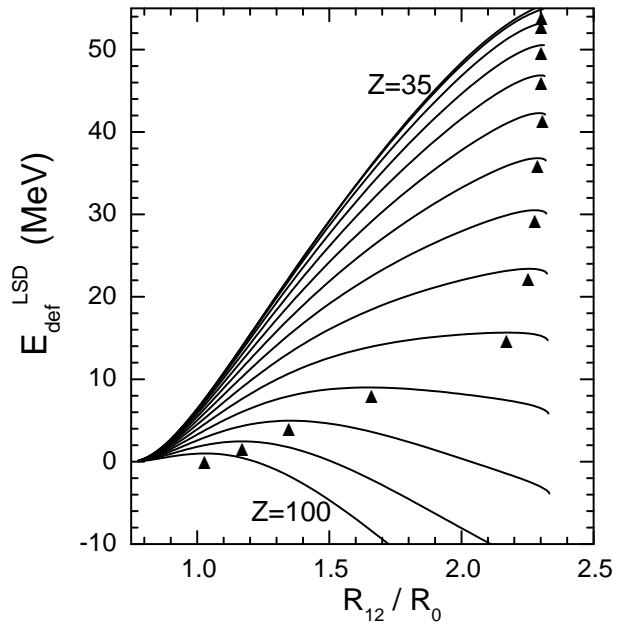

Fig. 7. LSD deformation energy (21) for the nuclei in the range $35 \leq Z \leq 100$, with $\Delta Z=5$, with fixed $(N-Z) / A=0.26$. The saddle point deformations are indicated by $\boldsymbol{\Lambda}$.

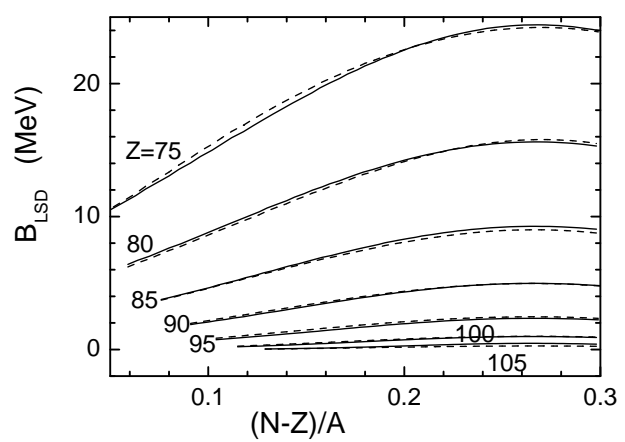

Fig. 8. LSD-fission barriers $B_{\mathrm{LSD}}$ (17)- (21) (solid line) as compared to the fit of Eq. (22) (dash line).

still accurate analytical approximation for $B_{\max }(Z), I_{0}(Z)$, $\Delta I(Z)$ in the whole region of $Z$. But it turned out possible to approximate $B_{\max }(Z), I_{0}(Z), \Delta I(Z)$ separately for $Z<75$ and $Z \geq 75$ by the polynomials

$$
\begin{aligned}
B_{\max }(Z) & \approx a_{0}+a_{1} Z+a_{2} Z^{2} * 10^{-2}+a_{3} Z^{3} * 10^{-4}, \\
I_{0}(Z) & \approx a_{4}+a_{5} Z * 10^{-4}, \\
\Delta I(Z) & \approx a_{6}+a_{7} Z * 10^{-2}+a_{8} Z^{2} * 10^{-4} .
\end{aligned}
$$

The coefficients $a_{n}$ are given in Table 1 . The quality of

Table 1. The coefficients $a_{n}$ of the approximation (23).

\begin{tabular}{|c|c|c|c|c|c|}
\hline & $a_{0}$ & $a_{1}$ & $a_{2}$ & $a_{3}$ & $a_{4}$ \\
\hline$Z<75$ & 23.92 & 2.235 & -3.816 & 1.128 & 0.2268 \\
\hline$Z \geq 75$ & 723.255 & -19.60 & 17.70 & -5.328 & 0.2739 \\
\hline & $a_{5}$ & $a_{6}$ & $a_{7}$ & $a_{8}$ & \\
\hline$Z<75$ & 4.340 & 0.7851 & -0.4141 & -0.438 & \\
\hline$Z \geq 75$ & -0.8633 & 0.09067 & 0.6911 & -0.661 & \\
\hline
\end{tabular}

approximation (23) is demonstrated in figure 8 . Though the coefficients $a_{n}$ look very different for $Z<75$ and $Z \geq 75$ the expressions (22) approximate the values of $B_{\max }, I_{0}$ and $\Delta I$ very accurately in the whole region of $Z$. The rms deviation of the approximation (22), (23) from the calculated fission barriers $B_{\mathrm{LSD}}$ for nuclei listed in tables of Möller $e t$ al [14] with $35 \leq Z \leq 105,0 \leq I \leq 0.3$ is about $150 \mathrm{keV}$, what is quite satisfactorily.
Within the macroscopic-microscopic approach the fission barrier height $V_{B}$ consists of contributions from the macroscopic and microscopic energies. In the case that the macroscopic energy is given by the LSD model, $V_{B}$ is

$$
V_{\mathrm{B}}=E_{\mathrm{LSD}}^{\text {(saddle) }}-E_{\mathrm{LSD}}^{\text {(g.s.) }}+\delta E^{\text {(saddle) }}-\delta E^{\text {(g.s.) }},
$$

where $\delta E \equiv \delta E_{\text {shell }}+\delta E_{\text {pair }}$ is the shell + pairing corrections.

According to the topographical theorem by Myers and Świątecki [10] the "barrier will be determined by a path that avoids positive shell effects and has no use for negative shell effects. Hence the saddle point energy will be close to what it would have been in the absence of shell effects, i.e., close to the value given by the macroscopic theory! "So, according to this topographical theorem the shell correction at the saddle can be neglected and the expression for the fission barrier heights takes the simple form

$$
V_{\mathrm{B}}=B_{\mathrm{LSD}}-E_{\text {micr }}^{\text {(g.s.) }} .
$$

Here, $B_{\mathrm{LSD}}=E_{\mathrm{LSD}}^{\text {(saddle) }}-E_{\mathrm{LSD}}^{(\mathrm{sph})}$ is the fission barrier height within the LSD-model, and $E_{\text {micr }}^{\text {(g.s. }}$ is given by (18). It is this quantity that is tabulated in [14]. Besides the ground state shell correction $\delta E^{\text {(g.s.) }}$, the $E_{\text {micr }}^{\text {(g.s.) }}$ contains the difference of liquid-drop energies taken at the ground state and at the spherical shape.

The ground state microscopic correction (18) is calculated and tabulated in Ref. [14] for all known and many yet unknown nuclei. For $B_{\mathrm{LSD}}$ an analytical approximation is obtained above. Thus, Eqs. (22)-(25) and Table 1 offer a very simple possibility to calculate the fission barrier for any nucleus.

The comparison of the fission barriers calculated in this way with the known experimental values is shown in figure 9.

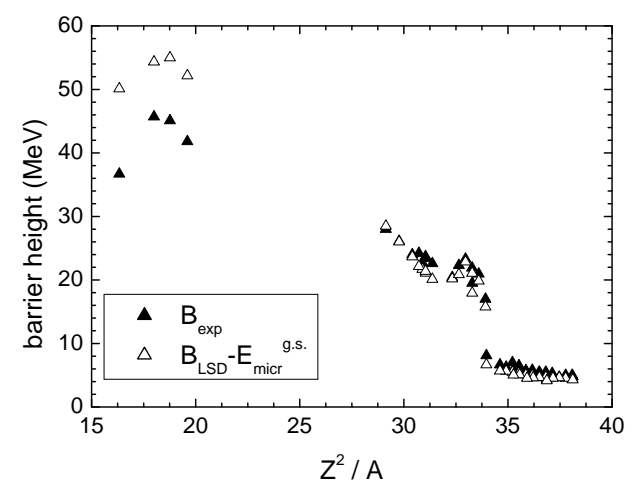

Fig. 9. Experimental $(\boldsymbol{\Delta})$ and calculated $(25)(\Delta)$ values of fission barrier heights.

The rms-deviation of the experimental and calculated values of the fission barrier heights for nuclei with $Z>70$ is $1.113 \mathrm{MeV}$. This is a little more than the rms-deviation obtained in [9] $(0.88 \mathrm{MeV})$. The main source of this difference is the use here of optimal shapes (14) which supply the lowest possible fission barriers. The difference between experimental and calculated values of the fission barriers for the four lightest nuclei with $35 \leq Z \leq 42$ is approximately the same as obtained in [9], i.e. of the order of $10 \mathrm{MeV}$. 
One can see in figure 9 that the calculated values of the fission barrier heights are predominantly lower as compared with the experimental values. This could be an indication that the shell correction at the barrier should not be ignored completely.

It was mentioned in [10] that the statement of the topographical theorem - that a macroscopic theory is accurate for saddle energies - becomes exact in the limit where the relative range of shell oscillations tends to zero. It is known from calculations of the potential energy surfaces that the wavelength of shell oscillations is not so small, but actually comparable with the width of the barrier. So, the $1.113 \mathrm{MeV}$ deviation of experimental versus calculated values of the fission barrier heights can, at least in part, be related to the use of the topographical theorem. Other uncertainties in determining the barrier heights may come from some model inaccuracies in the determining the ground-state microscopic correction [14] (the rms error of the ground state masses is $0.7 \mathrm{MeV}$ ). On the other hand, the topographical theorem offers a very simple and handy approximation (22)-(25) for the fission barriers.

Please, note that (25) is extremely simple to use. Except for the ground state correction which are taken from the tables, one only has to compute very simple analytic expressions (22 and (23).

\section{Summary}

The optimal shapes of fissioning nuclei are studied within the Lublin-Strasbourg Drop model [9]. The problem was solved by the method suggested by Strutinsky and collaborators $[1,7]$. In this method the shape of a nucleus is defined by minimization of the liquid drop energy with respect to the shape variation under the constraint that the volume and elongation are fixed. This method does not rely on any shape parameterization and provides the shapes of the lowest energy (optimal shapes).

The elongation of the nucleus calculated by this method is limited by some maximal deformation which is almost independent on the mass number. This critical deformation is interpreted as the scission point. The neck radius at critical deformation is rather large $(0.25-0.30) R_{0}$, i.e. the nucleus breaks apart not at zero but at finite neck.

The liquid drop fission barriers were calculated for isotopic chains in the range $35 \leq Z \leq 110$. It was found that, within a given chain, the maximal fission barrier heights occur roughly at $I=(N-Z) / A \approx 0.26$. A simple approximation for the liquid drop fission barriers heights containing only $Z$ and $I$ is obtained.

The topological theorem by Myers and Świątecki [10] was used to express the fission barrier height as the sum of the macroscopic barrier height and the ground state shell correction. This provides a very simple method to calculate fission barrier heights. Besides the ground state shell correction, which should be taken from the tables (e.g. in Ref. [14]), one only has to calculate simple analytical estimate for the liquid drop part of the barrier height.

The rms-deviation of calculated versus experimental values of fission-barrier heights for known nuclei with $Z \leq$ 70 is $1.113 \mathrm{MeV}$, a value which is comparable with the experimental uncertainties.

\section{References}

1. V. M. Strutinsky, N. Ya. Lyashchenko, N. A. Popov, Nucl. Phys. 46, 659 (1963)

2. N. Bohr and J. A. Wheeler, Phys. Rev. 56, 426 (1939)

3. V. V. Pashkevich, Nucl. Phys. A 169, 275 (1971)

4. F. A. Ivanyuk and K. Pomorski, Phys.Rev. C 79, 054327 (2009)

5. W. D. Myers and W. J. Świątecki, Ann. Phys. (N. Y.) 84, 186 (1974)

6. M. Brack, C. Guet, and H.-B. Hakansson, Phys. Rep. 123, 275 (1985)

7. V. M. Strutinsky, Zh. Exp. Theor. Fiz. 45, 1891 (1963)

8. H. v. Groote and E. Hilf, Nucl. Phys. A 129, 513 (1969)

9. K. Pomorski and J. Dudek, Phys. Rev. C 67, 044316 (2003)

10. W. D. Myers and W. J. Świątecki, Nucl. Phys. A 601, 141 (1996)

11. M. Antony, Nuclide Chart 2002, Impressions Francois, 103 Route de Marienthal, Haguenau, France; email address: ela.myelin@ires.in2p3.fr

12. W. D. Myers and W. J. Świątecki, Nucl. Phys. A 612, 249 (1997)

13. K. Pomorski and J. Dudek, Int. J. Mod. Phys. E 13, 107 (2004)

14. P. Möller, J. R. Nix, W. D. Myers, and W. J. Świa̧tecki, At. Data. Nucl. \& Data Tables 59, 249 (1995) 